\title{
Clinical, Laboratorial and Image Aspects of a Perforated Pyloric Ulcer Secondary to Nonsteroidal Anti-Inflammatory Use in a Cat
}

\author{
Tadeu Basualdo Junior, Ester Mercado Cedron Benetti, Julia Lanfredi Tonin, Márcio Virgílio Figueiredo da Silva, \\ Joyce Maira de Araújo \& Gabriel Utida Eguchi
}

\begin{abstract}
Background: Inappropriate use of drugs for veterinary patients represents a common problem at clinical practice. Nonsteroidal anti-inflammatories are one of these misused drugs and may lead to clinical status of challenging diagnosis. Adverse effects for patients submitted to its incorrect use may include simple cases such as pharmacological gastroenteritis to severe acute renal failure or perforated gastroenteric ulcers with no pathognomonic clinical signs. The objective of this report was to describe a case of a perforated pyloric ulcer secondary to prolonged use of meloxicam in a cat with its clinical, laboratorial and image aspects from the moment of suspicion until the diagnosis.

Case: An 8-year-old female feline was attended at the Veterinary Hospital of the Dom Bosco Catholic University, with main complaint being a mammary nodule with recent ulceration. Tumor staging and pre-surgical blood analysis were performed previous to total unilateral mastectomy. Eleven days post-surgery the patient was brought for suture removal, but it was observed stupor, moderate dehydration (estimated $10 \%$ ), $36.7^{\circ} \mathrm{C}$ rectal temperature, heart rate at 100 beats $/ \mathrm{min}$, respiratory rate at 60 breaths $/ \mathrm{min}, 40 \mathrm{mg} / \mathrm{dL}$ blood glucose, icterus and abdominal distension with tympany at percussion (fluid wave test was negative). Anamnesis revealed the possible use of meloxicam for 10 days. The first suspicion was sepsis, with enteric gas secondary to infection. Due to no classical signs of peritoneum effusion and possible severe enteric distension, abdominocentesis was not immediate performed. Complete blood count and serum biochemistry revealed a marked band leukocytosis associated with renal injury, supporting the first sepsis suspicion. Abdominal radiography revealed radiodensity of diffuse aspect at ventral topography but no evidence of marked enteric distension that would justify tympany. Abdominal ultrasound identified effusion predominantly hyperechogenic with hyperechogenic mesentery, indicative of peritonitis. A diagnostic abdominocentesis was performed revealing a dense yellow-green effusion with high suspicion of being gastroenteric liquid. Exploratory laparotomy was not authorized by the owner and the patient was submitted to euthanasia due to the bad prognosis. Macroscopic necropsy was performed and a perforated pyloric ulcer was identified along with an impregnated mesentery with a green-brown color (peritonitis), closing the diagnosis.

Discussion: The importance of reiteration regarding veterinary prescription orientation, especially for feline patients, is evidenced. Along the indiscriminate over-the-counter sale of veterinary drugs, self-medication prior to veterinary consultation is usual even for ongoing assisted patients. Considering the unspecific clinical signs that patients with perforated gastroenteric ulcers may present, the diagnosis may be challenging when no complementary image exams are immediate available. The stuporous mental state inhibiting possible manifestation of abdominal discomfort, absence of positive fluid wave test and tympany at percussion which prohibited a secure abdominocentesis could have led to a delay in diagnosis, if not for image support. Considering the emergency status of these patients, early diagnosis is crucial, therefore clinicians should have precaution when approaching patients with possible perforated gastroenteric ulcers and trust clinical history, even when classical signs of abdominal effusion are not present.
\end{abstract}

Keywords: abdominocentesis, NSAID, self-medication, surgery, symptomatology, tympany. 


\section{INTRODUCTION}

Inappropriate use of veterinarian drugs in Brazil is a common problem and includes prescriptions by unqualified people, home-made formulations and guided self-medication (when the owner reutilize previous prescriptions). Prospective epidemiology studies have identified $20 \%$ of self-medication report by owners for their pets, with the most commonly used drugs being antibiotics and anti-inflammatories [11].

Nonsteroidal anti-inflammatories (NSAIs) commercially available for pets, despite its cyclooxygenase-2 (COX-2) preferential inhibition, also affects COX-1 at some level [7]. Prolonged use of NSAIs may cause nonspecific clinical signs such as hypo or anorexia, emesis and diarrhea. These signs are usually consequences of gastroenteric erosions, ulcers or even acute renal injury [8]. Severe cases may evolve to a perforated ulcer, leading to an emergency case. Unfortunately, at clinical evaluation there are no pathognomonic signs that could close the case, therefore, it is essential a thorough anamnesis for an early diagnosis [10].

Classically, presence of abdominal pain and positive fluid wave test, indicative of abdominal effusion could lead the clinician for a secure diagnostic abdominocentesis. Without safe clinical parameters indicating peritoneal effusion, image complementary exams may aid the attendant [5].

Considering the emergency status of a perforated gastroenteric ulcer patient and variability of clinical signs that it could present, it is reported the clinical presentation, blood laboratorial results and image complementary exams of a feline with a perforated pyloric ulcer secondary to 10 days use of meloxicam.

\section{CASE}

It was attended at the Veterinary Hospital of the Dom Bosco Catholic University, Campo Grande, MS, Brazil, a feline patient, mixed breed, eight years old with main complaint being a mammary tumor (with inaccurate time of evolution period) with recent ulceration. Pre-surgical evaluation included a complete blood count (CBC), serum biochemistry (creatinine, urea and alanine aminotransferase), nodule cytology, chest radiograph and abdominal ultrasound. After, the patient was subjected to a routine unilateral total mastectomy and post-surgical prescription was as follows: cephalexin (Cefalexina ${ }^{\circledR} 30 \mathrm{mg} / \mathrm{kg} /$ every $12 \mathrm{~h}$ for
10 days) ${ }^{1}$, meloxicam (Maxicam ${ }^{\circledR} 0.1 \mathrm{mg} / \mathrm{kg} /$ every 24 $\mathrm{h}$ for 3 days) ${ }^{2}$, dipyrone (Dipirona ${ }^{\circledR} 25 \mathrm{mg} / \mathrm{kg} /$ every $12 \mathrm{~h} /$ for 5 days) ${ }^{3}$, tramadol chlorhydrate (Cloridrato de Tramadol ${ }^{\circledR} 2.5 \mathrm{mg} / \mathrm{kg} /$ every $12 \mathrm{~h}$ for 5 days $)^{4}$ and topical cleaning with sodium chloride solution $(0.9 \%)$ followed by topical antibacterial ointment (gentamicin, sulfanilamide, sulfadiazine, urea, vitamin A) [Vetaglos $^{\circledR}$ every $12 \mathrm{~h}$ until complete cicatrization $]^{5}$.

After 11 days post-surgery, the patient was brought exclusively for suture removal, but at physical examination it was observed stupor, moderate dehydration (estimated $10 \%), 36.7^{\circ} \mathrm{C}$ rectal temperature, heart rate at 100 beats/min, respiratory rate at 60 breaths/ $\mathrm{min}, 40 \mathrm{mg} / \mathrm{dL}$ blood glucose, icterus and abdominal distension with tympany at all sites of percussion (fluid wave test was negative). At anamnesis, it was revealed 10 days use of meloxicam.

Complete blood count, serum biochemistry and abdominal ultrasound and radiograph were performed. The comparative blood analysis (pre and post-surgery) are shown in Table 1.

Abdominal ultrasound revealed moderate repletion with free fluid in the peritoneal space, predominantly hyperechogenic and hyperechogenic mesentery, consistent with peritonitis images.

Abdominal radiograph showed an increase of radiopacity with diffuse aspect at ventral topography and fluid enteric content associated with moderate presence of gas (Figure 1).

After visualization of free fluid in the peritoneal space and confirmation of only a discrete distension of intestines, an abdominocentesis was performed (18 gauge catheter, retroumbilical) with immediate return of a yellow-green viscous effusion (1 mL) [Figure 2]. Due to the small volume acquired, a peritoneal lavage was performed respecting $20 \mathrm{~mL} / \mathrm{kg}$ of sterile isotonic saline solution (Solução fisiológica $0.9 \%{ }^{\circledR}$ ) 6 per cycle. The color of the liquid persisted after four cycles.

The patient was classified as an ASA V [1] and an exploratory laparotomy was indicated but not authorized by the owner. Due to the bad prognosis the patient was submitted to euthanasia and a macroscopic necropsy was then performed. The midline incision was followed by relief of a great volume of gas, in accordance with the tympany at abdominal percussion. The peritoneal space was filled with green aspect effusion and the mesentery was impregnated with a brown color tone and friable at manipulation. A perforated 
T. Basualdo Junior, E.M.C. Benetti, J.L. Tonin, et al. 2020. Clinical, Laboratorial and Image Aspects of a Perforated Pyloric Ulcer

ulcer $(0.5 \mathrm{~cm})$ with intense drainage of a dark viscous liquid was observed at the region of pylorus, confirming the diagnosis (Figure 3).

\section{DISCUSSION}

Clinical signs associated with perforated ulcers are unspecific and only with clinical evaluation the diagnosis may be difficult [3]. The first clinical suspicion for the animal of this report, considering the hypothermia, hypoglycemia, bradycardia, tachypnea and stupor was sepsis [2] due to a probable failure of the immune system secondary to undiagnosed infectious diseases such as feline immunodeficiency virus or feline leukemia virus. Feline infectious peritonitis was also considered, along with hepatic lipidosis associated

Table 1. Complete blood count and serum biochemistry pre and 11 days post-surgery of a feline patient diagnosed with a perforated pyloric ulcer.

\begin{tabular}{|c|c|c|c|}
\hline Complete blood count & Pre-surgery & Post-surgery & Reference values ${ }^{\mathrm{a}}$ \\
\hline Red blood cells $\left(10^{6} / \mu \mathrm{L}\right)$ & 3.86 & 2.87 & $5.5-7$ \\
\hline Hemoglobin (g/dL) & 5.9 & 4.6 & $8-16$ \\
\hline Hematocrit (\%) & 22.5 & 18.2 & $28-45$ \\
\hline Mean corpuscular volume (fL) & 58.29 & 63.41 & $39-55$ \\
\hline $\mathrm{MCHC}(\mathrm{g} / \mathrm{dL})$ & 26.22 & 25.27 & $31-35$ \\
\hline RDW - S.D. (fL) & 48.9 & 52.7 & $22.6-37.5$ \\
\hline RDW - C.V. (\%) & 17.8 & 17.4 & $13.1-20.5$ \\
\hline Icterus index (U.I.) & 2 & 100 & 2 \\
\hline Platelet count $\left(10^{3} / \mu \mathrm{L}\right)$ & 520 & 430 & $300-800$ \\
\hline White blood cells $\left(10^{3} / \mu \mathrm{L}\right)$ & 15.21 & 27.53 & $6-17$ \\
\hline Nt.SEG $(\%)$ & 74 & 70 & $35-75$ \\
\hline Nt.BAN (\%) & $--{ }^{b}$ & 7 & $0-3$ \\
\hline Lymphocyte (\%) & 16 & 18 & $22-55$ \\
\hline Monocyte (\%) & 8 & 5 & $1-4$ \\
\hline Eosinophil (\%) & 2 & --- & $2-12$ \\
\hline \multicolumn{4}{|l|}{ Serum biochemistry } \\
\hline Creatinine (mg/dL) & 1.5 & 3.16 & $0.8-1.8$ \\
\hline Urea (mg/dL) & 48 & 339 & $43-64$ \\
\hline $\operatorname{ALT}(\mathrm{UI} / \mathrm{L})$ & 32 & 22 & $10-80$ \\
\hline
\end{tabular}

MCHC: mean corpuscular hemoglobin concentration; RDW: red cell distribution width; Nt.SEG: segmented neutrophils; Nt.BAN: band neutrophils; ALT: alanine aminotransferase. ${ }^{\mathrm{a}}[6,9]$. ${ }^{\mathrm{b}} \mathrm{zero}$.

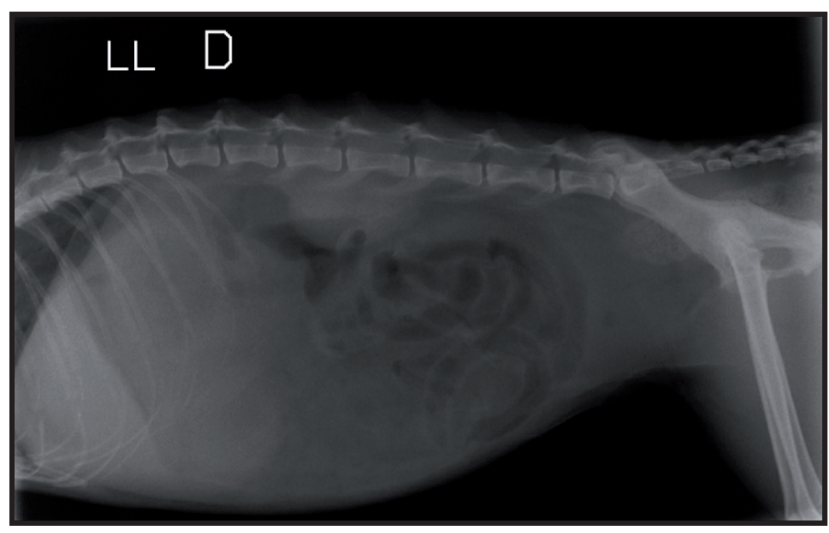

Figure 1. Abdominal radiograph of a feline patient with diagnosed perforated pyloric ulceration. Note the presence of radiopacity of diffuse aspect at ventral topography, indicative of effusion.

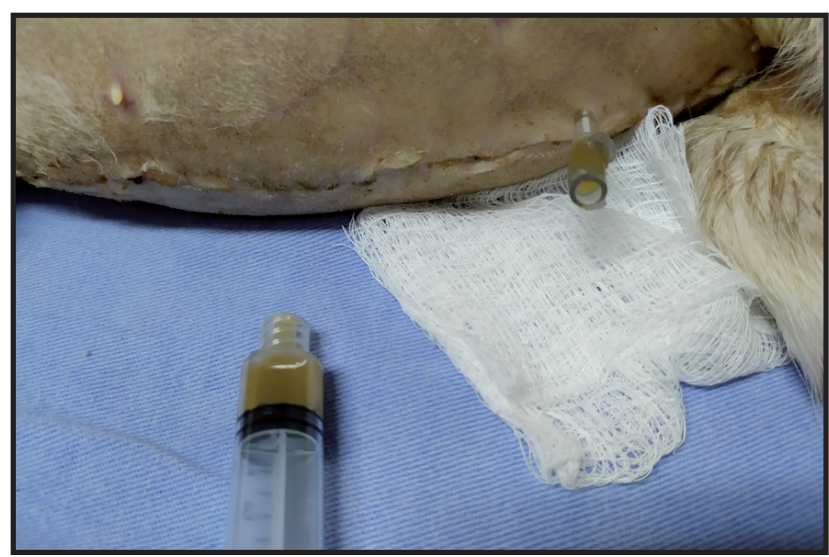

Figure 2. Effusion aspect at the diagnostic abdominocentesis of a feline patient with a diagnosed perforated pyloric ulcer. 


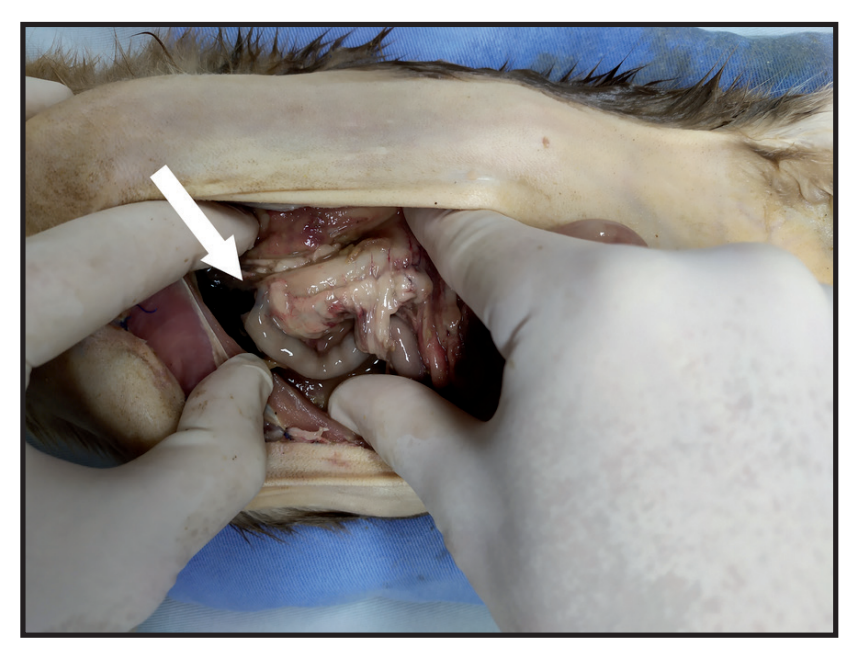

Figure 3. Perforated gastroenteric ulcer (arrow) at macroscopic necropsy of a feline patient submitted to 10 days use of meloxicam.

with hypovolemic shock due to a possible inefficient post-surgical nursing.

CBC analysis reinforced the suspicion of infection due to a marked band leukocytosis. Serum biochemistry also indicated the probability for sepsis, with a marked high concentration of creatinine and urea (Table 1).

The presence of gas in the peritoneal space probably interfered with a positive wave test, leading to a false impression of absence of effusion, which could had led to a diagnostic abdominocentesis prior to the ultrasound and radiographic exam. Considering the lack of easily available image exams out of commercial time, the clinician should take in to account all information the owner can provide, for that could be a key point for executing specific diagnostic procedures, even when the clinical presentation does not match the narrative.

It is also unusual the absence of emesis as complaint, but there are reports of veterinary patients with pyloric and duodenal lesions without gastroenteric signs [10]. Caution should be taken regarding the absence of complaints such as nausea or emesis for this patient, considering the inability of the owner in identifying the patient as sick.

If not the case of euthanasia, clinical intervention should include parameters goals such as temperature, serum glucose and volemia stabilization for a reasonable anesthetic security and exploratory surgery [4].
It is also important to reiterate the significance of further investigation and diagnosis even with an undesirable outcome. Despite the high suspicion of gastroenteric rupture considering the aspect of the abdominal effusion, diagnosis was only possible due to necropsy.

Even with all pre and post-surgical orientation that owners receive when attended by veterinarians, there are still misinterpretations regarding prescriptions, especially considering the variation of treatment duration depending on the pharmacological characteristics of the drug. For cats, that is particularly dangerous considering all peculiarities these patients have. While 10 days of meloxicam for dogs could not represent serious consequences, for cats that could rapidly evolve for death. It is a suspicion of the veterinarians involved in this case that the owner possibly misinterpreted the 10 days use of antibiotic as 10 days use of NSAI.

The case raised a new discussion and renovation regarding the individualization with which each orientation should be taken into account. Verbal reinforcement and text highlighting are the current tools being used, but new approaches are being considered, such as requisition for specific quantity sale of drugs for some cases.

In conclusion, clinicians should consider clinical history as guide for persistent investigation, even when clinical presentation does not match the narrative. Cats once more are proved as a sensitive species for NSAIs and care must be taken with the prescription of these pharmacological principles, either regarding the patient itself or for the prescription comprehension by the owner.

\section{MANUFACTURERS}

${ }^{1}$ EMS S/A. Hortolândia, SP, Brazil.

${ }^{2}$ Ouro Fino Saúde Animal. Cravinhos, SP, Brazil.

${ }^{3}$ Farmace Indústria Químico-Farmacêutica Cearense Ltda. Barbalha, CE, Brazil.

${ }^{4}$ Brainfarma Indústria Química e Farmacêutica S.A. Anápolis, GO, Brazil.

${ }^{5}$ Vetnil Indústria e Comércio de Produtos Veterinários Ltda. Louveira, SP, Brazil.

${ }^{6}$ JP Indústria Farmacêutica S.A. Ribeirão Preto, SP, Brazil.

Declaration of interest. The authors report no conflicts of interest. The authors alone are responsible for the content and writing of the paper. 


\section{REFERENCES}

1 American Society of Anesthesiologists (ASA). 2014. ASA Physical Status Classification System, 2p. Available at: $<$ https://www.asahq.org/standards-and-guidelines/asa-physical-status-classification-system >. [Accessed online in September 2019].

2 Brady C.A., Otto C.M., Van-Winkle T.J. \& King L.G. 2000. Severe sepsis in cats: 29 cases (1986-1998). Journal of the American Veterinary Medical Association. 217(4): 531-535.

3 Cariou M.P.L., Halfacree Z.J., Lee K.C.L. \& Baines S.J. 2010. Successful surgical management of spontaneous gastric perforations in three cats. Journal of Feline Medicine and Surgery. 12: 36-41.

4 Daure E., Ross L. \& Webster C.R.L. 2017. Gastroduodenal Ulceration in Small Animals: Part 1. Pathophysiology and Epidemiology. The Journal of the American Animal Hospital Association. 53(1): 1-10.

5 Ford R. \& Mazzaferro E.M. 2013. Procedimentos Diagnósticos e Terapêuticos: Procedimentos Avançados. In: Kirk \& Bistner Manual de Procedimentos Veterinários e Tratamento Emergencial. Rio de Janeiro: Elsevier, pp.786-787.

6 Kaneko J.J., Harvey J. \& Bruss M. 2008. Clinical Biochemistry of Domestic Animals. 6th edn. New York: Academic, 928 .

7 KuKanich B., Bidgood T. \& Knesl O. 2012. Clinical pharmacology of nonsteroidal anti-inflammatory drugs in dogs. Veterinary Anaesthesia and Analgesia. 39: 69-90.

8 Lucas G.N.C., Leitão A.C.C., Alencar R.L., Xavier R.M.F., Daher E.F. \& Silva Junior G.B. 2018. Pathophysiological aspects of nephropathy caused by non-steroidal anti-inflammatory drugs. Brazilian Journal of Nephrology. 41(1): 124-130.

9 Weiss D.J. \& Wardrop K.J. 2010. Schalm's Veterinary Hematology. 6th edn. Ames: Wiley-Blackwell, 1232p.

10 Wooten J.G., Lascelles B.D.X., Cook V.L., Law J.M. \& Blikslager A.T. 2010. Evaluation of the relationship between lesions in the gastroduodenal region and cyclooxygenase expression in clinically normal dogs. American Journal of Veterinary Research. 71(6): 630-636.

11 Zielke M., Carvalho L.F., Salame J.P., Barboza D.V., Gaspar L.F.J. \& Sampaio L.C.L. 2018. Avaliação do uso de fármacos em animais de companhia sem orientação profissional. Science and Animal Health. 6(1): 29-46. 